\title{
BMC Clinical Pathology reviewer acknowledgement 2014
}

Magdalena Morawska

\section{Contributing reviewers}

The editors of BMC Clinical Pathology would like to thank all of our reviewers who have contributed to the journal in volume 14 (2014).

Jackeline Agorreta

Spain

Martin Almquist

Sweden

Federico Ambrogi

Italy

Gabriel Arismendi-Morillo

Venezuela

Sylvia Asa

Canada

Amanjit Bal

India

Maria G. Barderas

Spain

Alfredo Berruti

Italy

Fay Betsou

Luxembourg

Thomas Bleck

USA

Miroslav Blumenberg

USA

Ferdinando Carlo Maria Cananzi Italy

Robert Canter

USA
Tai Chen

Yoon-La Choi South

Korea

Maurizio Colecchia

Italy

Mark Coles

UK

John Cooper

USA

Giuliana Cortese

Italy

Dajun Deng

China

Mohammad Derakhshan

UK

Marie-Caroline Dieu-Nosjean

France

Jurek Dobrucki

Poland

Stefan Eichmuller

Germany

Andreas Erbersdobler

Germany

Gavino Faa

Italy
Aurelie Fabre

Ireland

William Funk

USA

Jiri Gallo

Czech Republic

Jerad Gardner

USA

Jeff Garner

UK

Francesco Gelsomino

Italy

Paul Goodfellow

USA

Tomasz Gosiewski

Poland

Jessica Grieger

Australia

Maria Guembe

Spain

Christopher Hall

USA

Kim Henriksen

Denmark

Rui Henrique

Portugal

Correspondence: Magdalena.Morawska@biomedcentral.com

BioMed Central, Floor 6, 236 Gray's Inn Road, London WC1X 8HB, UK 
Gerard Hornstra

Netherlands

Paul Hruz

USA

John Hughes

UK

Issei Imoto

Japan

Beatrice Gabriela Ioan

Romania

Rosalyn Irby

USA

Mitsuaki Ishida

Japan

Hiroaki Ito

Japan

Kiseok Jang

South Korea

Jacques Jani

Belgium

Peter Jensen

Denmark

Carmen Jeronimo

Portugal

Kamisha Johnson-Davis

USA

Fabio Klamt

Brazil

Mirjana Kocova

Macedonia

John Koethe

USA

Lingquan Kong

China

Rachel Koshy

India

Leda Kovatsi

Greece

Larry Kricka

USA

Cord Langner

Austria

Mariana Leal

Brazil
Jinming $\mathrm{Li}$

China

Jose Antonio Lopez-Guerrero

Spain

Bertrand Ludes

France

Alexandre Manirakiza

Central African Republic

Federica Marchesi

Italy

Renato Mariani-Costantini

Italy

Vilma Martins

Brazil

Prashant Mathur

India

Fekicity Eb May

UK

Daniele Minardi

Italy

Hiroyuki Mineta

Japan

Glauco Miyahara

Brazil

Peter Molloy

Australia

Rodolfo Montironi

Italy

Sergio Morini

Italy

Mandi Murph

USA

Basavraj Nagoba

India

Luis Negrao

Portugal

Agnes Neuville

France

Alcina Nicol

Brazil

Krzysztof Okon

Poland

Ida Perrotta

Italy
Antoinette Perry

Ireland

Ruben Pio

Spain

Vito Pistoia

Italy

Peter Rambau

Tanzania

Khalil Razvi

UK

Christophe Redon

USA

Albert Reece

Australia

Andrea Renda

Italy

Richard Riedel

USA

Viviana Ritacco

Argentina

Ian Roberts

UK

Christoph Robier

Austria

Giulio Rossi

Italy

Felix Rückert

Germany

Ken Sakushima

Japan

Mario Scartozzi

Italy

Robert Schmidt

USA

Joel Schwartz

USA

Heidi Schwarzenbach

Germany

Nagamiah Selvakumar

India

Andrzej Semczuk

Poland

Shan-Rong Shi

USA 
Hiroyuki Shimada

USA

Mohamed Shoukri

Saudi Arabia

Sveinung Sorbye

Norway

Hugo Sousa

Portugal

Marla Steinbeck

USA

Christopher Stevenson

Australia

Simona Stolnicu

Romania

Walter Storkus

USA

Ying-Hsiu Su

USA

Michael Tachezy

Germany
Masahiro Tachi

Japan

Hua Tang

China

Emina Torlakovic

Canada

Chang-Yong Tsao

USA

Toyonori Tsuzuki

Japan

Deniz Tural

Turkey

Jeroen F. Vermeulen

Netherlands

Semir Vranic

Bosnia and Herzegovina

Jim Wiley

USA

Sabine Windhorst

Germany

\section{Kenneth Witwer}

USA

Hua-Sheng Xiao

China

Ke Zen

China

Ming Hao Zheng

Australia

Daniele Zink

Singapore

Received: 21 January 2015 Accepted: 21 January 2015 Published: 28 January 2015

doi:10.1186/1472-6890-15-1

Cite this article as: Morawska: BMC Clinical

Pathology reviewer acknowledgement 2014.

BMC Clinical Pathology 2015 15:1. 